\title{
Radon transform-based motion blurred silkworm pupa image restoration
}

\author{
Dan Tao, Zhengrong Wang*, Guanglin Li*, Guangying Qiu \\ (College of Engineering and Technology, Southwest University, Chongqing 400715, China)
}

\begin{abstract}
As for machine vision-based intelligent system in the application of discriminating and sorting the sex of silkworm pupae, the tail gonad was the unique physiological feature. However, motion blur, resulting from the live silkworm pupa's writhing motion at the moment of capturing image, could lose textures and structures (such as edge and tail gonad etc.) dramatically, which casted great challenges for sex identification. To increase the image quality and relieve the difficulty of discrimination caused by motion blur, an effective approach that including three stages was proposed in this work. In the image prediction stage, first sharp edges were acquired by using filtering techniques. Then the initial blur kernel was computed with Gaussian prior. The coarse version latent image was deconvoluted in the Fourier domain. In the kernel refinement stage, the Radon transform was applied to estimate the accurate kernel. In the final restoration step, a TV- $L_{1}$ deconvolution model was carried out to obtain a better result. The experimental results showed that benefiting from the prediction step and kernel refinement step, the kernel was more accurate and the recovered image contained much more textures. It revealed that the proposed method was useful in removing the motion blur. Furthermore, the method could also be applied to other fields.
\end{abstract}

Keywords: silkworm pupa, image restoration, radon transform, machine vision, motion blur, deblurring DOI: $10.25165 /$ j.ijabe.20191202.3681

Citation: Tao D, Wang Z R, Li G L, Qiu G Y. Radon transform-based motion blurred silkworm pupa image restoration. Int J Agric \& Biol Eng, 2019; 12(2): 152-159.

\section{Introduction}

Sericulture industry has big importance in the growth of economic and people's income. To acquire high quality silk, the cross-breeding silkworm pupae are adopted. Before breeding, it is necessary to separate the sex of silkworm pupae, which is accomplished manually with labor-costing and time-consuming ${ }^{[1]}$. Many studies have been reported to discriminate the sex of silkworm pupae. The study based on mass ${ }^{[2]}$ or shape just gave unsatisfied accuracy of $50 \%$. Other technologies, such as Magnetic resonance imaging $(\mathrm{MRI})^{[3]}$ or near infrared (NIR) spectroscopy ${ }^{[4]}$, were not suited for differentiating the sex of silkworm pupae because of the low generalization ability of model and the expense of the equipments. Kamtongdee et al. ${ }^{[5]}$ adopted the normalization cross-relation (NCC) method to classify the sex of silkworm pupae with accuracy of $88 \%$. Sumriddetchkajorn et al. ${ }^{[6]}$ developed an optical penetration-based system that containing complicated image processing steps (image thresholding, Blob filtering, image version and sex identification).

With recent rapid development of machine vision technology, image processing methods are utilized to identify silkworm pupae's sex. However, illumination, spatially-variant blur and noise in silkworm pupae image affect the sex discrimination accuracy of silkworm pupae.

Received data: 2017-07-29 Accepted data: 2019-01-06

Biographies: Dan Tao, PhD candidate, research interests: image restoration. Email: taodan_email@126.com; Guangying Qiu, Master, research interests: image processing, Email: qiuguangying602@163.com.

*Corresponding author: Zhengrong Wang, $\mathrm{PhD}$, research interests: artificial intelligence. Southwest University, Chongqing 400715, China. Email: wangxiong102@163.com, Tel: +86-13320267703; Guanglin Li, PhD, Professor, research interests: intelligent detection and mechatronics technology. College of Engineering and Technology, Southwest University, Chongqing 400715, China. Email: liguanglin@swu.edu.cn, Tel: +86-13883605913.
Our research group have already restored the noisy and low-illumination silkworm pupa image ${ }^{[7]}$ and removed the defocus blur from image due to limited depth of field ${ }^{[8]}$ in previous work. Nevertheless, the live silkworm pupa writhes when camera is gathering, which always brings motion blur, resulting in the tail gonad of silkworm pupae far from detectable, recognizable and difficult for retrieval. Hence, it was necessary to explore a novel method to remove motion blur from silkworm pupae image.

This work mainly focused on deblurring the image region including tail gonad that was similar with " $\mathrm{X}$ " pattern for female silkworm pupa or "dot" pattern for male one, as shown in red rectangle in Figure 1.

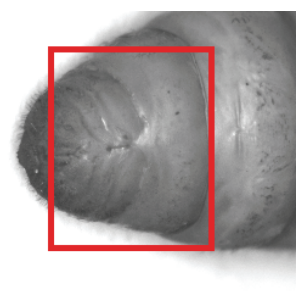

a. Female silkworm pupa

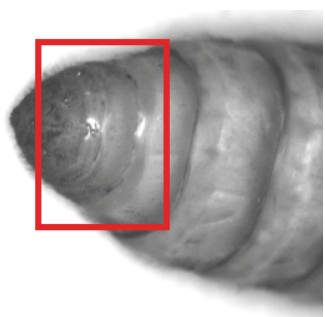

b. Male silkworm pupa
Figure 1 Tail gonad of silkworm pupa image

The motion blur can be modeled as the convolution of a latent image with a motion blur kernel when it is shift-invariant:

$$
B=k * I+n
$$

where, $B$ and $I$ respectively denotes the blurred image and latent image; $k$ is a motion blur kernel; $n$ denotes the additive noise and $*$ represents the convolution operator.

By using deconvolution operation, the latent image $I$ can be recovered. The difference between non-blind deconvolution and blind deconvolution is whether the kernel is known or not. In this paper, the silkworm pupae image restoration was a blind deconvolution problem that the kernel needed to be accurately estimated first. 
Some advances have been made to solve the blind deconvolution problem.

A. Blind deconvolution problem

The motion blur was assumed to be linear in some studies with the aim of decreasing the ill-posedness of blind deconvolution problem. Rav-Acha et al. ${ }^{[9]}$ recovered the latent image full of textures by adopting two images including horizontally blurred and vertically blurred. With the help of multiple input blurred images, Cho et al. ${ }^{[10]}$ solved the spatially-variant blur problem. For a single input, accurate kernel was calculated based on alpha matting by Dai and $\mathrm{Wu}^{[11]}$. $\mathrm{Ji}$ and $\mathrm{Liu}^{[12]}$ studied the problem of general types of blur (1D accelerated motion). When the motion blur became complex, these methods would be invalid.

Recent work mainly adopted the priors and multi-scale scheme. Fergus et al. ${ }^{[13]}$ fitted the heavy-tailed natural image prior by a mixture of Gaussians and the kernel was calculated based on a Variational Bayesian method. Shan et al. ${ }^{[14]}$ developed a unified model to estimate the latent image and the kernel. Xu et al. ${ }^{[15]}$ employed the $L_{0}$ gradient prior at the intermediate step of kernel estimation and the energy function was solved by a proximal method. Cho et al. ${ }^{[16]}$ computed the blur kernel using Radon transform in a MAP framework. Pan et al. ${ }^{[17]}$ designed a prior including $L_{0}$ regularized intensity and gradient and a half-quadratic splitting strategy was applied to iteratively estimate the latent image. Levin et al. ${ }^{[18]}$ thought that the MAP method favored the trivial solution.

By predicting the explicit edges from the input blurred image, accurate kernel was estimated without using priors. Joshi et al. ${ }^{[19]}$ predicted the sharp edges by propagating the local intensity toward the edges. Cho et al. ${ }^{[20]}$ acquired sharp edges by adopting filtering techniques. These methods imposed computation efficient Gaussian priors, resulting in the un-accurate kernel.

When input blurred image contained noise, Tai et al. ${ }^{[21]}$ denoised and deblurred the image jointly in a unified scheme. Zhong et al. ${ }^{[22]}$ estimated the kernel from filtered images based on radon transform. By combing the $L_{1}$ fidelity term and Mumford-Shah regularizer term together, the latent image was effectively recovered ${ }^{[23]}$. Joshi et al. ${ }^{[24]}$ incorporated a local two-color prior to decrease noise.

B. Non-blind deconvolution problem

When the kernel was known, deconvolution were accomplished based on Richardon-Lucy (RL) or Weiner filtering, which were sensitive to noise and resulted in ringing and artifacts. Yuan et al. ${ }^{[25]}$ proposed an improved edge-preserving bilateral Richardon-Lucy (BRL) method to render a better result.

A key characteristic of non-blind deconvultion method was the priors. The $L_{1}$ prior enforced the sparsity of image and led to an efficient solver. Other priors, such as total variation regularization term, were also explored ${ }^{[26-29]}$.

\section{Silkworm pupa image restoration}

Compared with the classical blind deconvolution problem, silkworm pupa image deblurring had its own distinctive characteristics. It was important to recover the tail gonad region of silkworm pupa image, which determined the sexclassification performance. The challenges of this paper mainly contained three parts,

1) The image captured by the camera included the whole silkworm pupa. But the tail gonad region just occupied a small part of the whole image, which had insufficient information to estimate the kernel, increasing the difficulties to restore latent tail gonad region image.

2) Due to the writhing during capturing the silkworm pupa image, the edge information was degraded severely and image analysis such as edge detection were hard to accomplish. The methods using large scale edges would fail.

3) Because the edges in the silkworm pupa image were simple (horizontal or vertical), the methods based on isotropy assumption ${ }^{[30]}$ were not suited for silkworm pupa image restoration.

Here, the blur of the silkworm pupa image was assumed uniform (spatially invariant). The aim was to recover the latent silkworm pupa image from the blurred input, with confidence that the restored image could be classified by the intelligent system effortlessly.

Specifically, this paper made following contributions,

1) Effective filtering techniques, i.e. bilateral filtering and shock filtering, were employed to recover sharp edges from the original image. The initial kernel estimation and coarse version latent image were respectively obtained by Gaussian prior and $L_{2}$ gradient prior.

2) During kernel estimation process, radon transform was incorporated with the MAP framework to iteratively estimate the kernel and the intermediate latent image using the iterative reweight least squares (IRLS) approach.

3) In the final latent image restoration step, the TV- $L_{1}$ scheme was used and optimized based on half-quadratic splitting algorithm.

The rest of the paper was organized as follows. The mathematical models were provided in Section 2. Benefiting from prediction step, the initial kernel and the coarse latent image could be recovered. In the kernel estimation step, with the initial kernel propagated from the prediction step, accurate kernel was obtained. Simulated experiments and real experiments were shown in Section 3. Section 4 presented the conclusions.

\section{Proposed approach}

Our algorithm mainly contained three steps including prediction step, kernel estimation step, latent image restoration step. The flow chart was shown in Figure 2.

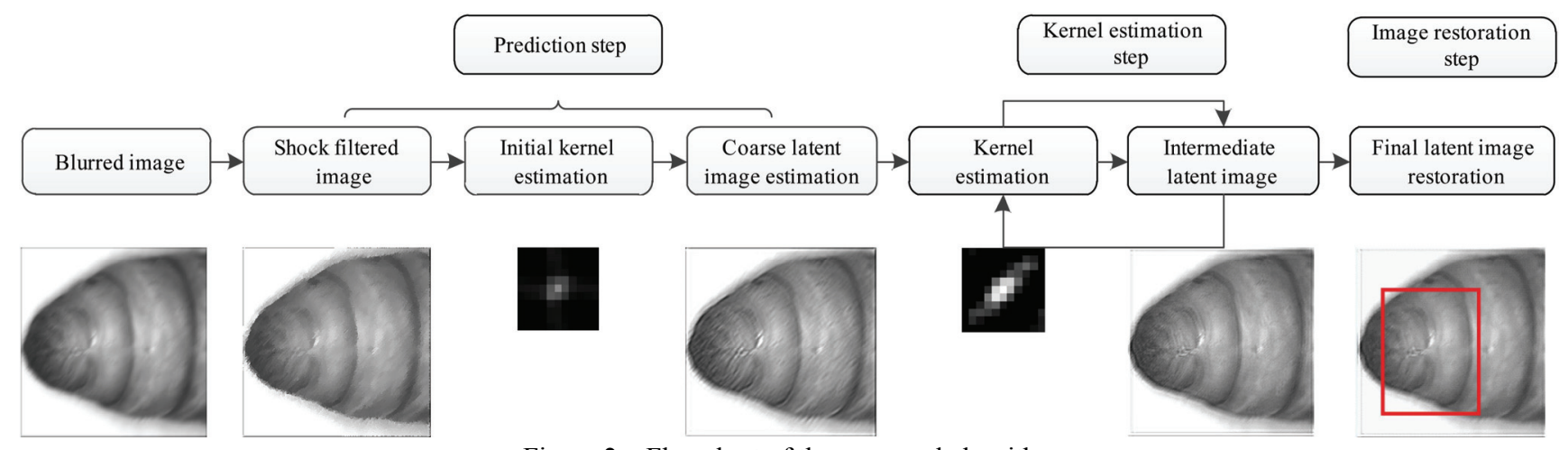

Figure 2 Flowchart of the proposed algorithm 


\subsection{Prediction processing}

The prediction step consisted of sharp edges restoration, kernel initialization, and coarse image reconstruction.

\subsubsection{Sharp edge recovering}

Like other motion deblurring methods, the blurred image was first filtered to acquire the salient edges. After bilateral filtering, shock filtering was carried out to enhance the image features and obtain sharp edges ${ }^{[31]}$. The equation of shock filter is expressed as follows:

$$
I_{t+1}^{s}=I_{t}^{s}-\operatorname{sign}\left(\Delta I_{t}^{s}\right)\left\|\nabla I_{t}^{s}\right\| d t, I_{0}=B i l \otimes B
$$

where, $I_{t}^{s}$ denotes an image at time $t ; \Delta I_{t}^{s}$ and $\nabla I_{t}^{s}$ respectively represents the Laplacian and gradient of $I_{t}^{s}, d t$ is the time step for a single evolution; and we set $d t=1$. Bil denotes the bilateral filtering operator. The initial value of $I_{t}^{s}$ is $I_{0}$ that smoothed using bilateral filtering.

The shock filtered image had little noise and included sharp edges after pre-processing, as shown in Figure 3.
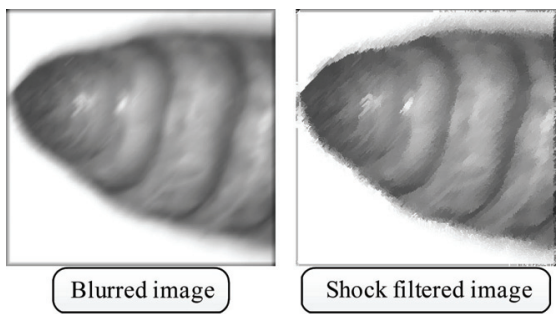

Figure 3 Shock filtered silkworm pupa image

\subsubsection{Initial kernel estimation}

With sharp edges from the shock filtered image $I^{s}$ in section 2.1.1, initial kernel was computed quickly with the Gaussian regularization term. The objective function is formulated as follows $^{[20]}$ :

$$
E(k)=\left\|k * \nabla I^{s}-\nabla B\right\|_{2}^{2}+\gamma\|k\|_{2}^{2}
$$

where, $\gamma$ is served as weight between the regularization term and the data fidelity term. The solution of equation (3) was obtained by performing Fast Fourier transform (FFT) and setting the derivative w.r.t $k$ to zero ${ }^{[32]}$, as shown in equation (4):

$$
k=F^{-1}\left(\frac{F\left(\partial_{x} I^{s}\right)^{*} F\left(\partial_{x} B\right)+F\left(\partial_{y} I^{s}\right)^{*} F\left(\partial_{y} B\right)}{F\left(\partial_{x} I^{s}\right)^{2}+F\left(\partial_{y} I^{s}\right)^{2}+\gamma}\right)
$$

where, $F^{-1}(\cdot)$ and $F(\cdot)$ represent inverse FFT and FFT operation respectively; $F(\cdot)^{*}$ denotes the complex conjugate operator; $\partial x$ and $\partial y$ are the partial derivatives in two directions, respectively.

\subsubsection{Coarse version image estimation}

With the estimated $k$ from the Section 2.1.2, the gradient prior $\|\nabla I\|$ was added to the energy function ${ }^{[20]}$, was shown in equation (5):

$$
E(I)=\|k * I-B\|_{2}^{2}+\zeta\|\nabla I\|_{2}^{2}
$$

where, $\zeta$ is a regularization parameter, and in this paper $\zeta=0.01$. According to the reference ${ }^{[32]}$, Equation (5) had the closed-form solution:

$$
I=F^{-1}\left(\frac{F(k)^{*} F(B)}{F(k)^{*} F(k)+\zeta\left(F(\partial x)^{*} F(\partial x)+F(\partial y)^{*} F(\partial y)\right)}\right)
$$

The restored image $I$ had well preserved sharp edges that could benefit the accurate kernel estimation.

The algorithm was sketched in algorithm 1 .

\section{Algorithm 1. Prediction processing}

Inputs: Input image $B, d t, \zeta, \gamma$.

For $d=1 \rightarrow 5$ do
(1) Pre-processing the input blurred image by using bilateral filtering and shock filtering techniques (Equation (2)).

(2) Estimating the initial kernel with $L_{2}$ regularization term (Equation (4)).

(3) Estimating the latent image with the gradient prior (Equation (6)). end for

Outputs: Initial kernel $k$ and the coarse latent image $I$.

\subsection{Accurate kernel estimation}

\subsubsection{The radon transform of blur kernel}

The radon transform of blur kernel $k$ was the collection of integrals $k(x, y)$ along straight lines which could be denoted using offset $\rho$ and the orientation $\theta$, as presented in follows ${ }^{[16]}$.

$$
\Phi_{\theta}^{k}(\rho)=\int_{-\infty}^{+\infty} \int_{-\infty}^{+\infty} k(x, y) \delta(\rho-x \cos (\theta)-y \sin (\theta)) d x d y
$$

where, $\Phi_{\theta}^{k}(\rho)$ denotes the projections of $k$ along the direction orthogonal to different orientation $\theta$. If the projections of $k$ were sufficient, original kernel $k$ could be estimated by adopting the inverse operation of radon transform.

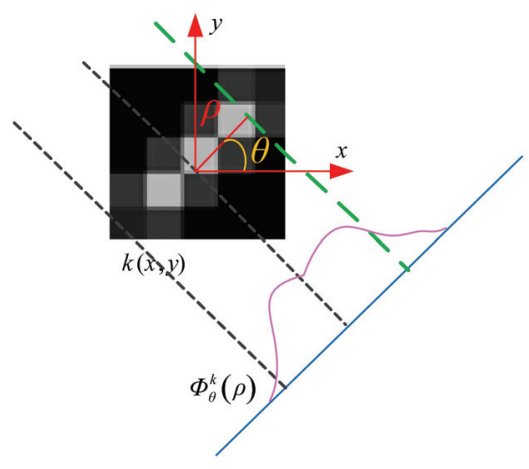

Figure 4 Radon transform

The ideal step edge of input image $B$ was defined as $H(x, y)$, which was 1 if $x \geq 0$, or 0 otherwise. The blurred step edge was denoted as $B_{H}=k * H$. By taking the derivative of step edge along the direction orthogonal to the edges, it produced a line, i.e. $B_{l}=\frac{\partial B_{H}}{\partial x}=k * \delta_{x} \quad\left(\right.$ here $\delta_{x}(x, y)=\delta(x)$ ). The $y$ was omitted since the formula did not depend on it. By expanding $k(x) * \delta(x)$, we got $B_{l}(x)=\int k(u) \delta(x-u) d u$. Comparing with the Equation (7), when $\theta=0$, the vertical projection $\Phi_{0}^{k}(x)$ of $k$ was equal to $B_{l}(x)^{[16]}$.

From above analyses, the step edge's first derivative $B_{l}$ was the radon projections $\Phi_{0}^{k}$ along the direction $\theta$ orthogonal to the step edges. In order to reconstruct an accurate kernel $k$, the projections should be aligned: the center of objection among all kernel projections should be the same. We shifted the projections such that the center of mass in each projection was in the middle ${ }^{[16]}$.

\subsubsection{Radon projections from the blurred image}

In order to detect step edges from input image $B$, an edge map was built with edge detection. The stable edges with two features were chosen. First, selected edges should have a high contrast guaranteeing that the ratio of edge profile and noise was high. The other was that the blurred edge profiles were not contaminated by adjacent edges. If blurred line profile was enough, the kernel projections could be computed by taking orthogonal slice to the blurred line.

\subsubsection{Kernel estimation in MAP scheme}

Having estimated the projections of kernel $k$, based on the Bayesian formulation, we sought to maximize posterior probability of blur kernel $k$ and intermediate latent image $I$ jointly ${ }^{[16]}$,

$$
(\hat{k}, \hat{I})=\arg \max _{k, I} p(k, I \mid B) \propto p(B \mid(k, I)) p(k) p(I)
$$

where, $p(B \mid k, I)$ is the likelihood term; $p(k)$ and $p(I)$ are kernel prior 
and image prior, respectively. $\quad p(B \mid k, I)$ is often modeled by using the observation model (Equation (1) $)^{[16]}$,

$$
p(B \mid k, I) \propto \exp \left(-\frac{\|B-k * I\|^{2}}{2 \omega_{e}^{2}}\right)
$$

where, $\omega_{e}^{2}$ is the variance of image noise. Leven et al. ${ }^{[33]}$ argued that the joint solution of blur kernel and latent image could not produce a satisfactory result, usually leading to a trivial solution when used the Equation (9) as the likelihood term. To solve the problem, the projections of $k$ were augmented to the likelihood term based on the constraint that the explicit projections should match its estimated projections ${ }^{[16]}$.

$$
p(B \mid k, I) \propto \exp \left(-\frac{\|B-k * I\|^{2}}{2 \omega_{e}^{2}}-\frac{1}{2 \omega_{e e}^{2}} \sum_{i=1}^{M}\left\|\Phi_{i}-R_{\theta_{i}} k\right\|^{2}\right)
$$

where, $i$ indexes edge samples; $M$ is the number of radon projections; $\omega_{e e}^{2}$ is the variance of observed noise; $\Phi_{i}$ is the measured projection; $R_{\theta_{i}}$ is the a projection operator along the $i t h$ sample's domain orientation $\theta_{i}$.

The knowledge about the intensity profiles of blur kernel was used to model the kernel prior $p(k)$,

$$
p(k) \propto \exp \left(-\varepsilon_{1}\|k\|^{0.9}\right)
$$

where, $\varepsilon_{1}=1.5$ in this paper

About the image prior, a regularization gradient operator was adopted,

$$
p(I)=r_{1}\|\nabla I\|^{2}
$$

Then, the Equation (8) was modified as below,

$$
p(k, I \mid B) \propto \exp
$$

$$
\left(-\left(\frac{\|B-k * I\|^{2}}{2 \omega_{e}^{2}}+\frac{1}{2 \omega_{e e}^{2}} \sum_{i=1}^{M}\left\|\Phi_{i}-R_{\theta_{i}} k\right\|^{2}+\varepsilon_{1}\|k\|^{0.9}+r_{1}\|\nabla I\|^{2}\right)\right)
$$

The problem to estimate $I$ was simplified using a Gaussian distribution with initial input $k^{[16]}$.

$$
E(I)=\arg \min _{I} \frac{\|B-k * I\|^{2}}{2 \omega_{e}^{2}}+r_{1}\|\nabla I\|^{2}
$$

The optimal solution to Equation (14) was obtained in frequency domain.

$$
I=F^{-1}\left(\frac{\frac{F(k)^{*} F(B)}{2 \omega_{e}^{2}}}{\frac{F(k)^{*} F(k)}{2 \omega_{e}^{2}}+r_{1}\left(F(\partial x)^{*} F(\partial x)+F(\partial y)^{*} F(\partial y)\right)}\right)
$$

Then it reduced to a least square problem to estimate $k$ using the latent image.

$$
E(k)=\arg \min _{k} \frac{\|B-k * I\|^{2}}{2 \omega_{e}^{2}}+\frac{1}{2 \omega_{e e}^{2}} \sum_{i=1}^{M}\left\|\Phi_{i}-R_{\theta_{i}} k\right\|^{2}+\varepsilon_{1}\|k\|^{0.9}
$$

The Equation (16) was nonconvex and usually was solved using an iterative re-weighted least squares process (LRLS) ${ }^{[26]}$.

The pseudocode of kernel estimation was shown in Algorithm 2.

Algorithm 2. Kernel estimation algorithm

Inputs: The coarse latent image estimated in Section 2.1.3 above as the input

blurred image, initial kernel, $\varepsilon_{1}, r_{1}$.

1. Detecting the step edges from the blurred image.

2. Estimating the Radon projections $\Phi^{k}{ }_{\theta}$ of the kernel $k$ along the direction $\theta$ orthogonal to the step edges by taking the first derivative of the step edge.

For $d=1 \rightarrow 5$ do

Estimating $I$ using Equation (15).

Estimating $k$ using Equation (16).

end for

Outputs: Accurate blur kernel $k$, intermediate latent image $I$.

\subsection{Final latent image restoration}

After acquiring kernel $k$ from the Section 2.2, the next step was deconvolution. To produce a satisfied result, a fast and efficient $\mathrm{TV}-L_{1}$ deconvolution model was introduced ${ }^{[29]}$.

$$
\min _{I} \sum_{j}^{n^{2}} \lambda_{j}\left\|\nabla_{j} I\right\|_{2}+\|k * I-B\|_{1}
$$

where, $n \times n$ and $\lambda_{j}$ were respectively image size and weight parameter, $\nabla_{j} I$ denoted the discrete gradient operation at pixel $j$, where $j$ was the image index. For the regularization term and the fidelity term, the penalties were non-linear. By adopting the half-quadratic splitting technique, the solution could be obtained.

For each pixel, a variable $z$ was introduced to equal the $k * I-B$, and $w_{j}=\left(\left(w_{j}\right)_{x},\left(w_{j}\right)_{y}\right)$ was used to denote the gradient $\nabla_{j} I=\left(\left(\nabla_{j} I\right)_{x}\right.$, $\left(\nabla_{j} I_{y}\right)$ in the horizontal and vertical direction. The Equation (17) was modified as follows ${ }^{[29]}$ :

$$
\min _{z, w, I} \lambda\|w\|_{2}+\beta\|w-\nabla I\|_{2}^{2}+\|z\|_{1}+\eta\|z-(k * I-B)\|_{2}^{2}
$$

When $\beta \rightarrow \infty$ and $\eta \rightarrow \infty$, the solution of Equation (18) approximated to that of Equation (17). By fixing two variables among $z, w, I$, it was easier to minimize the third one. Since $w$ and $z$ were decoupled, they were estimated independently.

The values of $z$ and $w$ were initialized to zeros. During each iteration process, the solution of $I$ was obtained by solving:

$$
\min _{I} \beta\|w-\nabla I\|_{2}^{2}+\eta\|z-(k * I-B)\|_{2}^{2}
$$

It was obvious that Equation (19) could be solved in a closed form. FFT was applied to both sides of Equation (19).

$$
\begin{aligned}
& F(I)\left[F(\partial x)^{*} F(\partial x)+F(\partial y)^{*} F(\partial y)+\frac{\eta}{\beta} F(k)^{*} F(k)\right]= \\
& F(\partial x)^{*} F\left(w_{x}\right)+F(\partial y)^{*} F\left(w_{y}\right)+\frac{\eta}{\beta} F(k)^{*}[F(B)+F(z)]
\end{aligned}
$$

where, $\beta \neq 0$. The inverse FFT is applied to $F(I)$.

$$
I=F^{-1}\left(\frac{F(\partial x)^{*} F\left(w_{x}\right)+F\left(\partial_{y}\right)^{*} F\left(w_{y}\right)+\frac{\eta}{\beta} F(k)^{*}[F(B)+F(z)]}{F(\partial x)^{*} F(\partial x)+F(\partial y)^{*} F(\partial y)+\frac{\eta}{\beta} F(k)^{*} F(k)}\right)
$$

For a fixed $I$ and $z$, minimizing Equation (18) for $w$ was to solve the following problem.

$$
\min _{w} \lambda\|w\|_{2}+\beta\|w-\nabla I\|_{2}^{2}
$$

The solution of $w$ could be obtained by the two-dimensional shrinkage formula ${ }^{[34]}$,

$$
w=\max \left\{\|\nabla I\|-\frac{\lambda}{\beta}, 0\right\} \frac{\nabla I}{\|\nabla I\|}
$$

For a fixed $I$ and $w$, minimizing Equation (18) for $z$ was equivalent to solving:

$$
\min _{z}\|z\|_{1}+\eta\|z-(k * I-B)\|_{2}^{2}
$$

Computing $z$ became an easier one-dimensional shrinkage problem $^{[29]}$,

$$
z=\max \left(\|k * I-B\|-\frac{1}{\eta}, 0\right) \operatorname{osgn}(k * I-B)
$$

where, ${ }^{\circ}$ and $\operatorname{sgn}(\cdot)$ represented the point-wise product and the sign function. $\quad \eta \neq 0$. About the parameter setting, we employed the start-warm scheme ${ }^{[14,34]}$. The iterations here began with small penalties $\left(\eta_{0}=1, \beta_{0}=2 e^{-2}\right)$ and stopped with the optimal number of iteration steps and accuracy. It was noted that the maximum parameters were set to $100, \eta_{\max }=\beta_{\max }=100$. Usually, it only used 
less than 12 iterations to converge in the experiments.

Algorithm 3 showed the pseudocode for the image restoration.

Algorithm 3: Final image restoration

Input: Blurred image $B$ and the accurate kernel $k$.

$I \leftarrow B, \eta \leftarrow \eta_{0}$.

Repeat

Solve for $z$ using Equation (25).

$\beta \leftarrow \beta_{0}$

Repeat

Solve for $w$ using Equation (23).

Solve for $I$ in the frequency domain using Equation (21)

$\beta \leftarrow 2 \beta$.

Until $\beta>\beta_{\max }$

$\eta \leftarrow 2 \eta$

Until $\eta>\eta_{\max }$

Output: Latent image $I$

\section{Experimental results}

The proposed method was robust to the choice of parameters by using the same settings $\gamma=2$ (in Equation (3)), $\varepsilon_{1}=1.5$ (in Equation (12)), $r_{1}=0.5$ (in Equation (22)) in this paper. The results of four methods, i.e., Xu et al. $(2010)^{[15]}$, Pan et al. $(2014)^{[17]}$, Cho et al.(2011) ${ }^{[16]}$ and Cho et al. (2009) ${ }^{[20]}$ were compared with the proposed method. All the experiment results were implemented on a computer with Intel i3-3240, 3.40GHz, memory $4 \mathrm{~GB}$, and 64 bit operation system, matlab2012a and $\mathrm{VC}++6.0$.

Structural similarity $(\mathrm{SSIM})^{[35]}$ and peak signal-to-noise ratio $(\mathrm{PSNR})^{[35]}$ are very effective techniques to assess the image quality.

$$
\begin{gathered}
\mathrm{PSNR}=10 \log \left(\frac{255^{2} \times n}{\|\hat{I}-I\|_{2}^{2}}\right) \\
\mathrm{SSIM}=\frac{\left(2 \mu_{\hat{I}} \mu_{I}+C_{1}\right)\left(2 \sigma_{\hat{I}} \sigma_{I}+C_{2}\right)}{\left(\mu_{\hat{I}}^{2}+\mu_{I}^{2}+C_{1}\right)\left(\sigma_{\hat{I}}^{2}+\sigma_{I}^{2}+C_{2}\right)}
\end{gathered}
$$

where, $\hat{I}$ and $I$ respectively denote the original latent image and the reconstructed image. $n \times n$ is the image size in Equation (26). In Equation (27); $\mu_{\hat{I}}, \sigma_{\hat{I}}$ and $\mu_{I}, \sigma_{I}$ are respectively mean,

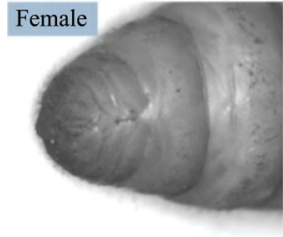

a. Original sharp image

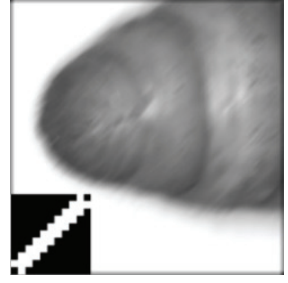

b. Synthetic image with applied kernel with size of $11 \times 11$

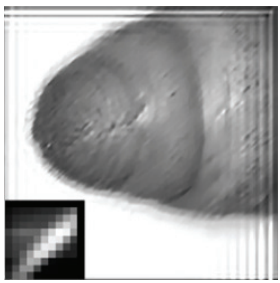

c. Xu et al. $(2010)^{\lfloor 15\rfloor}$ variance of gray density of the original latent and reconstructed silkworm pupa image. $C_{1}=\left(q_{1} G\right)^{2}$ and $C_{2}=\left(q_{2} G\right)^{2}$ (here, $q_{1}$ and $q_{2}$ are small constant, $G$ is the dynamic range of the pixel values (255 for 8-bit grayscale images) ) are constant for guaranteeing stable computation of SSIM.

The accuracy of kernel estimation is effectively assessed by using a kernel similarity metric, i.e., normalized cross-correlation $^{[36]}$

$$
S(k, \hat{k})=\max _{\varepsilon_{2}} R\left(k, \hat{k}, \varepsilon_{2}\right)
$$

where, $k$ and $\hat{k}$ respectively represents the estimated kernel and the truth kernel, $\varepsilon_{2}$ and $R(\cdot)$ respectively denote the possible shift between these two kernels and the normalized cross-correlation function. $R(\cdot)$ was shown as follows,

$$
R\left(k, \hat{k}, \varepsilon_{2}\right)=\frac{\sum_{\tau} k(\tau) \cdot \hat{k}\left(\tau+\varepsilon_{2}\right)}{\|k\| \cdot\|\hat{k}\|}
$$

where, $\tau$ represents the element coordinates; $\|\cdot\|$ is 2-norm. The larger kernel similarity metric value is, the more accurate the estimated kernel is.

The kernel estimation accuracy of simulated data experiments was evaluated by kernel similarity and the quality of restored images were evaluated by PSNR, SSIM. The higher the values of PSNR and SSIM were, the better the quality of restored image was.

\subsection{Simulated data experiments}

The simulated data containing one female silkworm pupa image and one male silkworm pupa image with the size of $256 \times 256$ were generated by adding motion blur with motion parameters (length 13 , angle 45 ) to the original silkworm pupa image. The kernel size was usually selected from $11 \times 11,13 \times 13,15 \times 15$.

The results of different methods were presented in Figure 5. Figure $5 \mathrm{a}$ was the original clear images, Figure $5 \mathrm{~b}-5 \mathrm{f}$ were restored images by $\mathrm{Xu}$ et al. $(2010)^{[15]}$, Cho et al. (2009) ${ }^{[20]}$, Cho et al. $(2011)^{[16]}$, and this study, respectively. The estimated kernels were displayed as insets. PSNR, SSIM and kernel similarity metric were shown in Table 1 and Table 2, respectively.
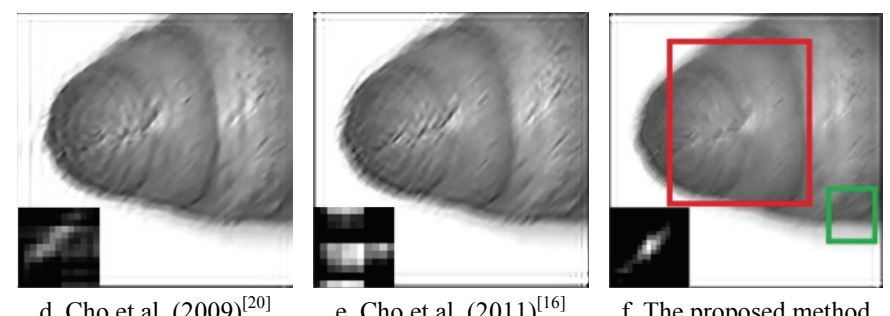

d. Cho et al. (2009) $)^{\lfloor 20\rfloor}$

\begin{tabular}{|c|c|c|c|c|c|}
\hline Silkworm pupa image & Assessment Index & $\mathrm{Xu}$ et al. $(2010)^{[15]}$ & Cho et al. $(2009)^{[20]}$ & Cho et al. $(2011)^{[16]}$ & The proposed method \\
\hline \multirow{2}{*}{ Male } & $\operatorname{PSNR}(\mathrm{db})$ & 15.924 & 16.919 & 16.253 & 17.126 \\
\hline & SSIM & 0.734 & 0.858 & 0.711 & 0.869 \\
\hline \multirow{2}{*}{ Female } & $\operatorname{PSNR}(\mathrm{db})$ & 16.075 & 16.784 & 16.486 & 17.011 \\
\hline & SSIM & 0.755 & 0.767 & 0.704 & 0.822 \\
\hline
\end{tabular}

f. The proposed method

Figure 5 Simulated experiments

Table 1 Quantitative comparison on the synthetic examples of simulated experiments

Table 2 Kernel similarities of simulated experiments

\begin{tabular}{ccccc}
\hline $\begin{array}{c}\text { Silkworm pupa } \\
\text { image }\end{array}$ & $\begin{array}{c}\text { Xu et al. } \\
(2010)^{[15]}\end{array}$ & $\begin{array}{c}\text { Cho et al. } \\
(2009)^{[20]}\end{array}$ & $\begin{array}{c}\text { Cho et al. } \\
(2011)^{[16]}\end{array}$ & $\begin{array}{c}\text { The proposed } \\
\text { method }\end{array}$ \\
\hline Male & 0.688 & 0.739 & 0.544 & $\mathbf{0 . 7 8 3}$ \\
Female & 0.767 & 0.676 & 0.427 & $\mathbf{0 . 7 8 1}$ \\
\hline
\end{tabular}

In terms of visual quality, the proposed method gave the best performance by preserving the edges and reducing artifacts, and improved the silkworm pupa image from unrecognizable to recognizable to some extent. Due to lack of prediction step, the results of Cho et al. (2011) $)^{[16]}$ contained much blurs and only a fraction of details were restored. Although Cho et al. (2009) ${ }^{[20]}$ 
recovered many textures around the tail gonad region, the distortion was severe and the recovered texture was unnatural. The results of Xu et al. (2010) ${ }^{[15]}$ could restore many image textures, but server ringing artifacts still existed in the edges, especially in the tail of the silkworm pupa image. At the same time, the prior performance of the proposed method could be quantitatively explained by the PSNR and SSIM presented in Table 1. It was shown that PSNR values and SSIM values of the proposed method were the highest, which manifested the proposed method could successfully reconstruct blurred degraded observation as the original latent image.

In addition, the sparse shape of the estimated kernel was close to the truth, which benefited from the kernel-sparsity prior in section 2.2.3, while other methods' results possibly produced a dense kernel. At the same time, the accuracy of kernels could be quantitatively explained by kernel similarity values presented in Table 2. It showed that the proposed method's values were larger than other methods, which testified the proposed method really had an impact on estimating the blur kernel. Thus, the proposed method was effectively in removing motion blur.

However, the heavy blur in the green rectangle could not be removed. Usually, the blind deblurring method was difficult to recover the entire information. Fortunately, the green rectangle region as mentioned above was not the focus of identification, because the tail gonad in the red rectangle region guaranteed the success of identification.

\subsection{Real data experiments}

In this section, one real data set (including one female silkworm pupa image and one male silkworm pupa image with size of $256 \times 256$ ), was employed for surveying the performance of the proposed method. The kernel size was usually selected from $7 \times 7$, $9 \times 9,11 \times 11,13 \times 13$.

The results were displayed in Figure 6. Figure 6a was the original blurred image, Figures 6b-6e were the results from Pan et al., Cho et al., Cho et al. and the proposed method, respectively.

Among these results, the proposed method could more effectively remove blur, and preserve the sharp edges, reducing the ringing artifacts around the tail gonad. Benefiting from the gradient and intensity prior, Pan et al. (2014) ${ }^{[17]}$ method recovered a large fraction of textures, but the results still suffered much blur. The results of Cho et al. (2011) ${ }^{[16]}$ were close to that of Cho et al. $(2009)^{[20]}$ that image textures were effectively restored, while sever ringing artifacts still existed.
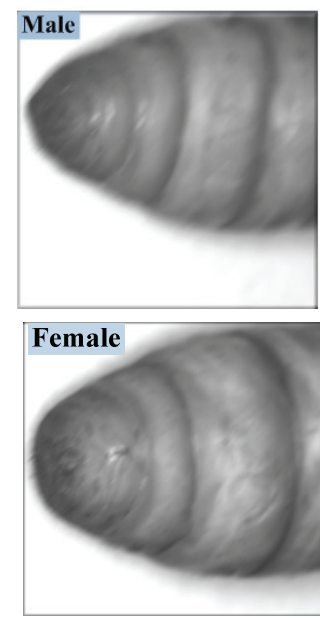

a. Real blurred image
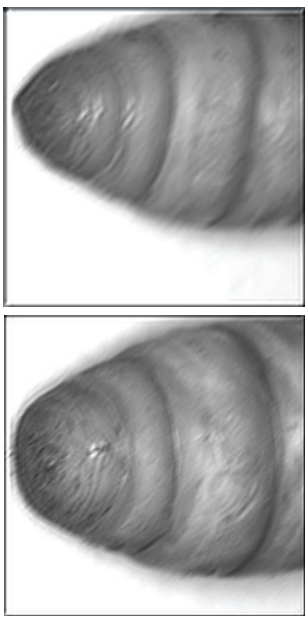

b. Pan et al. $(2014)^{[17]}$

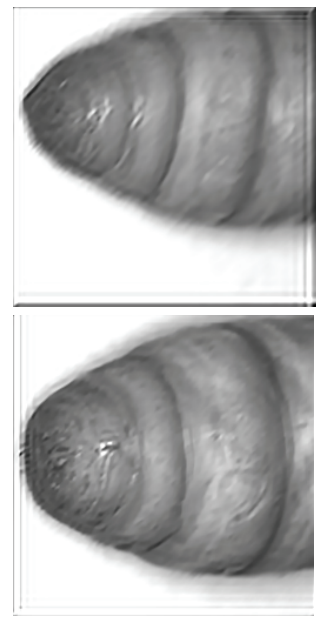

c. Cho et al. (2009) $)^{[20]}$

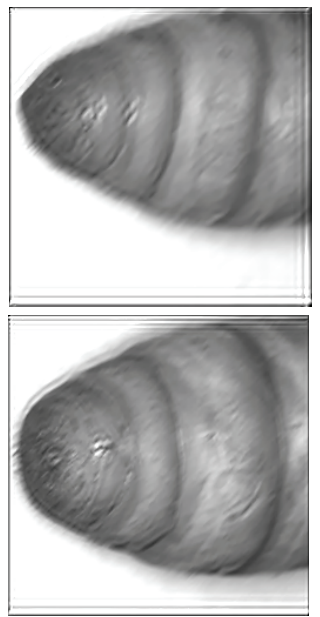

d. Cho et al. $(2011)^{[16]}$

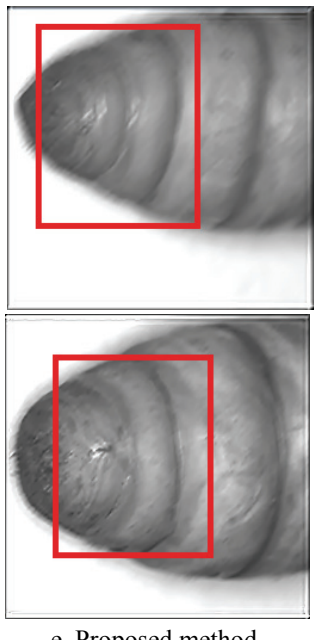

e. Proposed method

Figure 6 Real data experimental results

\subsection{Robustness with variation of motion blur intensity experiments}

Some experiments were conducted to discuss the robustness, as shown in Figure 7. A set of original blurred silkworm pupa image with size of $256 \times 256$ was utilized, containing one female silkworm pupa image and one male silkworm pupa image. The slighter blur image was applied with motion blur parameter (length 7 , angle 45), the heavier blur image was applied with motion blur kernel parameter (length 11, angle 45). The kernel size was specified from $7 \times 7,9 \times 9,11 \times 11,13 \times 13,15 \times 15$.

To a certain extent, the proposed method eliminated motion blur, increased image textures and sharpness contrast. Along with the blur of silkworm pupa images increasing, the proposed method could effectively restore many textures, while other methods could not produce satisfactory results with severe artifacts and blurred edges. Accordingly, relatively, it was un-obstructive to assert that the proposed method was some robust against the motion blur.

\subsection{Sex identification experiment}

The sex identification experiment of silkworm pupae based on
Hough transform were carried out to assess the deblurring performance on the other hand ${ }^{[16]}$. First, the template maps of female and male silkworm pupae were respectively built by computing the geometric and textural features based on Hough transform. Then the feature map was built by selecting the same size region as the template map in the unrecognized image from the start to end gradually. Finally, the matching score was computed by voting. The score indicated the similarity. If the score was larger than 0.5 , the sex could be identified successfully. There were totally 300 silkworm pupae and the results were drawn in Table 3. Obviously, the recognition rate improved after image restoration, which indicated that the proposed method effectivly restored the blurred image and improved the sex identification accuracy.

Table 3 Silkworm pupa sex recognition results

\begin{tabular}{ccc}
\hline & Recognized sum & Recognized rate/\% \\
\hline Before restoration & 136 & 45.3 \\
After restoration & 263 & 87.6 \\
\hline
\end{tabular}



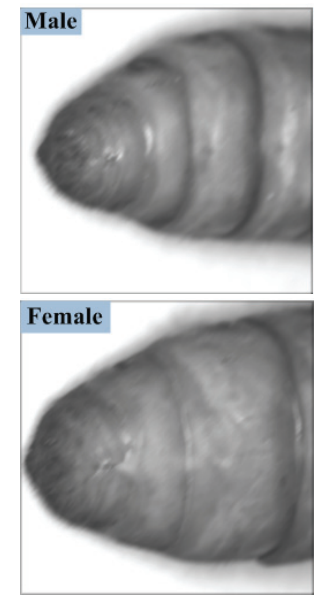

a. Blurred image applied with motion blur kernel parameter (length 7, angle 45)
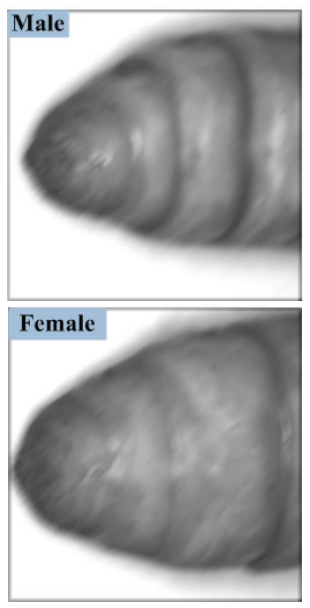

f. Blurred image applied with motion blur kernel parameter (length 11, angle 45)

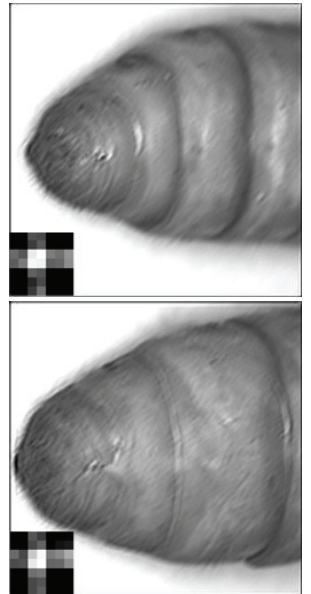

b. Pan et al. $(2014)^{[17]}$
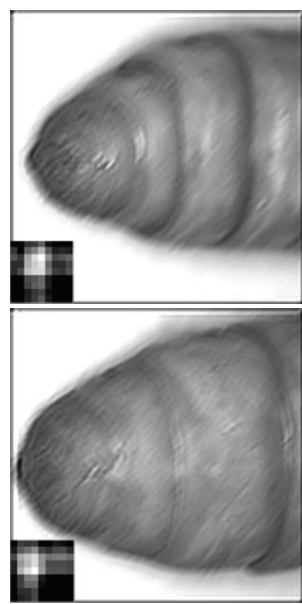

g. Pan et al. $(2014)^{[17]}$
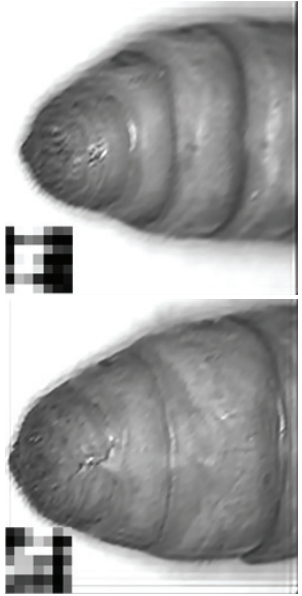

c. Cho et al. $(2009)^{[20]}$
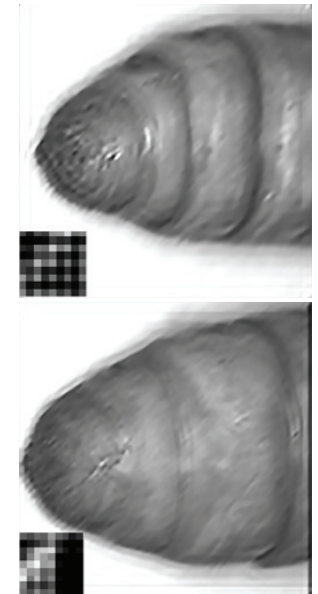

h. Cho et al. (2009) ${ }^{\lfloor 20\rfloor}$

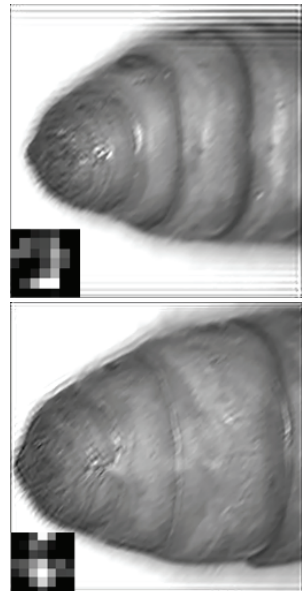

d. Cho et al. $(2011)^{[16]}$
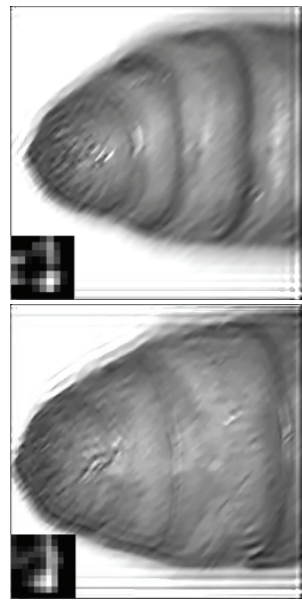

i. Cho et al. $(2011)^{\lfloor 16\rfloor}$
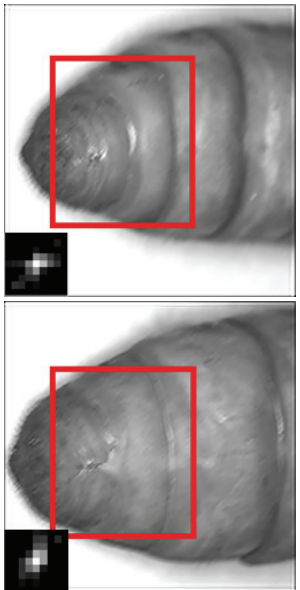

e. The proposed method
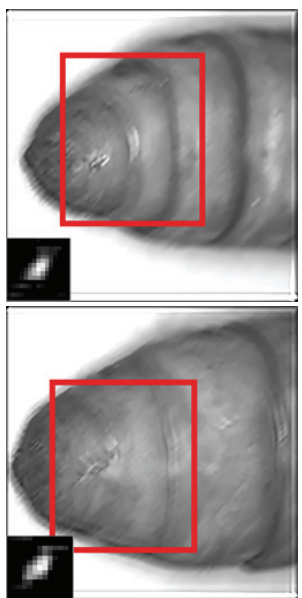

j. The proposed method

Figure 7 Robust experiments

\section{Conclusions}

To improve the sex identification accuracy of silkworm pupae when motion blur was involved, a three stage strategy that containing prediction step, kernel estimation step and latent image restoration was employed. Benefiting from the prediction step based on the global information and the coarse-to-fine framework, the latent silkworm pupa image was recovered without ringing artifacts and edge preserving.

It was notable that there were still many questions remained in blind motion deconvolution. Our proposed method performed well in the case of uniform blurring over the silkworm pupa image, but, it may fail in other conditions, such as saturated pixels from strong lights, sever noise and potential spatially-vary blur.

\section{Acknowledgements}

The authors would like to thank the editors and anonymous reviewers for their valuable suggestions. The research was financially supported by Chongqing Science and Technology Commission Projects under Grant No. cstc2013yykfA80015 and Grant No. cstc2017shms-xdny80080, Fundamental Research Funds for the Central Universities under Grant No.XDJK2016A007, XDJK2018D011, Doctoral Scientific Research Foundation of Southwest University Project Grant No. SWU114109.

\section{[References]}

[1] Jordan J, Ellington J, McCoy J. An electronic version system for sorting cotten boll worm pupae by sex. IEEE Conf. Signals, Syst. Comput, 1899; 2: 538-542.

[2] Seo Y, Morishima H, Hosokawa A. Separation of male and female silkworm pupae by weight: prediction of separability. The Japanese Society of Agricultural Machinery, 1985; 47: 191-195.

[3] Liu C, Ren Z H, Wang H Z, Yang P Q, Zhang X L. Analysis on Sex of Silkworms by MRI Technology. International Conference on Biomedical Engineering and Informatics, 2008; 2: 8-12.

[4] Jin T, Liu L, Tang X, Chen H. Differentiation of male, female and dead silkworms while in the cocoon by near infrared spectroscopy. Journal of Near Infrared Spectroscopy, 1995; 3: 89-95.

[5] Kamtongdee C, Sumriddetchkajorn S, Sa-Ngiamsak C. Feasibility study of silkworm pupa sex identification with pattern matching. Computers \& Electronics in Agriculture, 2013; 95(1): 31-37.

[6] Sumriddetchkajorn S, Kamtongdee C. Optical penetration-based silkworm pupa sex sensor structure. Applied optics, 2012; 51(4): 408-412.

[7] Tao D, Li G L, Wang Z R, Qiu G Y. Algorithm and experiments of noisy low-illumination silkworm pupa images restoration. Transactions of the CASE, 2015; 31(15): 147-152. (in Chinese)

[8] Tao D, Li G L, Wang Z R, Qiu G Y. Silkworm pupa image restoration based on aliasing resolving algorithm and identifying male and female. Transactions of the CASE, 2016; 32(16): 168-174. (in Chinese)

[9] Rav-Acha A, Peleg S. Two motion-blurred images are better than one. Pattern Recognition Letters, 2005; 26(3): 311-317.

[10] Cho S, Matsushita Y, Lee S. Removing Non-Uniform Motion Blur from Images. IEEE International Conference on Computer Vision, 2007; 1-8. 
[11] Dai S, Wu Y. Motion from blur. In Proc. CVPR, 2008; 1-8.

[12] Ji H, Liu C. Motion blur identification from image gradients. IEEE Conference on Computer Vision and Pattern Recognition, 2008; 1-8.

[13] Fergus R, Singh B, Hertzmann A, Roweis S T, Freeman W T. Removing camera shake from a single photograph. in Proc. SIGGRAPH, 2006; 25(3): 787-794.

[14] Shan Q, Jia J Y, Agarwala A. High-quality motion deblurring from a single image. ACM Transactions on Graphics, 2008; 27(3): 1-10.

[15] Xu L, Jia J Y. Two-phase kernel estimation for robust motion deblurring. ECCV, 2010; 4: 157-170.

[16] Cho T S, Paris S, Horn B K P, Freeman W T. Blur kernel estimation using the radon transform. CVPR, 2011; 42: 241-248.

[17] Pan J S, Hu Z, Su Z X, Yang M-H. Deblurring text images via L0-regularized intensity and gradient prior. IEEE Conference on Computer Vision and Pattern Recognition (CVPR 2014), 2014; 2901-2908.

[18] Levin A, Weiss Y, Durand F, Freeman W T. Understanding and evaluating blind deconvolution algorithms. CVPR, 2009; 8: 1964-1971.

[19] Joshi N, Szeliski R, Kriegman D. PSF estimation using sharp edge prediction. CVPR, 2008; 1-8.

[20] Cho S, Lee S. Fast motion deblurring. ACM Transactions on Graphics, 2009; 28(5): 1-8.

[21] Tai Y W, Lin S. Motion-aware noise filtering for deblurring of noisy and blurry images. CVPR, 2012; 157: 17-24.

[22] Zhong L, Cho S, Metaxas D, Paris S, Wang J. Handling noise in single image deblurring using directional filters. CVPR, 2013; 9: 612-619.

[23] Bar L, Sochen N, Kiryati N. Image deblurring in the presence of salt-and-pepper noise. Scale Space and PDE Methods in Computer Vision. Springer Berlin Heidelberg, 2005; 3459: 107-118.

[24] Joshi N, Zitnick C L, Szeliski R, Kriegman D J. Image deblurring and denoising using color priors. CVPR, 2009; 1550-1557.

[25] Yuan L, Sun J, Quan L, Shum H Y. Progressive inter-scale and intra-scale non-blind image deconvolution. ACM SIGGRAPH, 2008;
27(3): 1-10.

[26] Levin A, Fergus R, Durand F, Freeman W T. Image and depth from a conventional camera with a coded aperture. ACM Transactions on Graphics, 2007; 26(3): 1-9.

[27] Wang Y, Yang J, Yin W, Zhang Y. A new alternating minimization algorithm for total variation image reconstruction. Siam Journal on Imaging Sciences, 2008; 1(3): 248-272.

[28] Krishnan D, Fergus R. Fast image deconvolution using hyper-Laplacian priors. International Conference on Neural Information Processing Systems. Curran Associates Inc, 2009; 1033-1041.

[29] Yang J, Zhang Y, Yin W. An efficient TV-L1 algorithm for deblurring multichannel images corrupted by impulsive noise. Siam Journal on Scientific Computing, 2009; 31(4): 2842-2865.

[30] Wang C, Yue Y, Dong F, Tao Y, Ma X, Clapworthy G, et al Nonedge-specific adaptive scheme for highly robust blind motion deblurring of natural imagess. IEEE Trans Image Process, 2013; 22, 884-897.

[31] Osher S, Rudin L I. Feature-oriented image enhancement using shock filters. Siam Journal on Numerical Analysis 1990; 27(4): 919-940.

[32] Bertsekas D P, Nedic A, Ozdaglar A E. Convex analysis and optimization athena scientific. Journal of Mathematical Analysis \& Applications, 2003; 129(2): 420-432.

[33] Levin A, Weiss Y. User assisted separation of reflections from a single image using a sparsity prior. IEEE Transactions on Pattern Analysis and Machine Intelligence, 2004; 29(9): 1647-1654.

[34] Yang J, Yin W, Zhang Y, Wang Y. A fast algorithm for edge-preserving variational multichannel image restoration. Siam Journal on Imaging Sciences, 2009; 2(1): 569-592.

[35] Wang Z, Bovik A C, Sheikh H R, Simoncelli E P. Image quality assessment: from error visibility to structural similarity. IEEE Transactions on Image Processing 2004; 13: 600-612.

[36] $\mathrm{Hu} \mathrm{Z}$, Yang M H. Learning good regions to Deblur. International Journal of Computer Vision, 2015; 115(3): 345-362. 